\title{
CLOSED FORM INTEGRATION OF THE ROTATING PLANE PENDULUM NONLINEAR EQUATION ${ }^{\dagger}$
}

\author{
GIOVANNI MINGARI SCARPELLO AND DANIELE RITELLI
}

\begin{abstract}
The article deals with the nonlinear differential equation of the frictionless motion of a heavy pendulum swinging in a vertical plane which rotates at a fixed angular speed. The authors focused on its closed form integration by means of the Jacobi elliptic functions. This research took its origin by an autonomous work of the authors; this subject was also developed by [3], who did a treatment by far different from ours.
\end{abstract}

\section{Introduction}

In this paper we will perform the explicit integration - through the Jacobian elliptic functions - of the nonlinear scalar ordinary differential equation:

$$
\left\{\begin{array}{l}
\ddot{\vartheta}=a \sin \vartheta \cos \vartheta-b \sin \vartheta, \quad a, b>0 \\
\vartheta(0)=\vartheta_{0} \\
\dot{\vartheta}(0)=\dot{\vartheta}_{0}
\end{array}\right.
$$

The problem (1.1), has been originated by the Watt (flyball) governor motion analysis, in the absence of both, the friction along the round and the feedback on the angular speed $\omega$ of the driving diametral shaft. This problem is present in the nonlinear literature, [4] and [3]: it belongs to the direct dynamical ones.

We are then dealing with the frictionless circular (radius $R$ ) motion of a fixed length bead whose oscillating plane rotates about the circle's diameter joining its poles at a fixed angular speed $\omega$. Let $\vartheta$ be the mobile point coordinate. The point speed component tangent to the trajectory in its plane is $R \dot{\vartheta}$, that on the weight direction is $R \omega \sin \vartheta$.

Received January 24, 2002; revised October 11, 2002.

2000 Mathematics Subject Classification. Primary. 70H06; Secondary. 33E05, 34A05, 34C25.

Key words and phrases. Nonlinear differential equations, Watt governor, Jacobi elliptic functions, Weierstraß theorem.

$\dagger$ Research supported by MURST grant: Metodi matematici in economia 


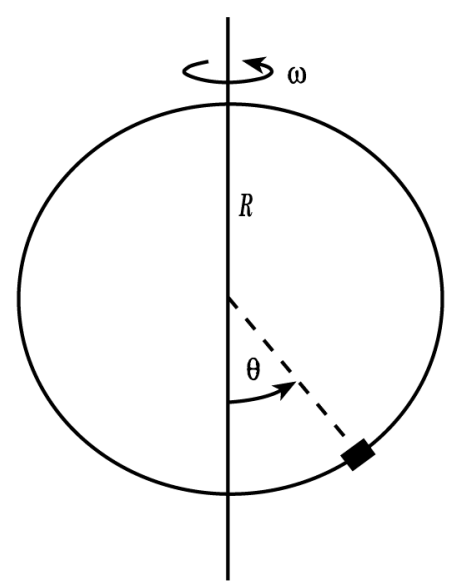

The system is hamiltonian, holonomic and single degree of freedom. Taking as origin the circle's center, and as reference the rotation axis oriented downwards, with the $\vartheta$ angle measured according to the picture, the potential, due to the weight only, will be $m R g \cos \vartheta$. The Lagrange function is:

$$
\mathcal{L}=\frac{1}{2} m R^{2}\left(\dot{\vartheta}^{2}+\omega^{2} \sin ^{2} \vartheta\right)+m R g \cos \vartheta
$$

which will lead us to the relative motion Lagrange equation:

$$
\ddot{\vartheta}=\omega^{2} \sin \vartheta \cos \vartheta-\frac{g}{R} \sin \vartheta
$$

The equation (1.1) then is a generalized pendulum equation whose motion plane is rotating at a constant angular speed. The (1.2) bifurcations have been analyzed at [4], pages $40-42$, which the reader, interested in the motion's qualitative analysis, is referred to. The article [3] analyzes a similar physical object (but its hamiltonian is perturbed from the reference one) and their relevant phase curves, in order to detect stability and resonance.

Our overall different pattern of enquiry is the response of $(1.2)$ on the $(\vartheta, t)$ plane for a large span of initial conditions, keeping the hamiltonian function unperturbed, and disregarding instability and resonances. The Legendre reduction of the elliptic integrals to their standard form will be followed according to the Bateman Project Staff work [9].

The (1.1) structure refers to the Weierstraß theorem: we are faced with a second order autonomous differential equation:

$$
\left\{\begin{array}{l}
\ddot{\vartheta}=f(\vartheta) \\
\vartheta(0)=\vartheta_{0}, \\
\dot{\vartheta}(0)=\dot{\vartheta}_{0},
\end{array}\right.
$$


for which, separating the variables, the time equation, is:

$$
t=\int_{\vartheta_{0}}^{\vartheta} \frac{d \xi}{\sqrt{\Phi(\xi)}}
$$

with:

$$
\Phi(\vartheta)=2 \int_{\vartheta_{0}}^{\vartheta} f(\xi) d \xi+\dot{\vartheta}_{0}^{2}
$$

and where the sign either + or - has to be selected, according to the sign of the initial speed $\dot{\vartheta}_{0}$, or, if $\dot{\vartheta}_{0}=0$ accordingly with $f\left(\vartheta_{0}\right)$ sign, as it is well known, see e.g. [7] page 114. The $\Phi=0$ roots' existence and nature, marks completely the motion, deciding its periodicity or aperiodicity. Furthermore the reality condition $\Phi \geq 0$ must be met. For details the reader is referred to [1] pages 287-293, or to [7] page 114 .

In next section, we will tackle the case of zero initial speed (akinetic case) with the angle $\vartheta_{0}$ in the first quadrant, whilst at section 3 the integration of the motion equation will be carried out. Section 4 will go into the motion of non zero starting speed (kinetic case), to be integrated in section 5. The system dynamical behaviour is ruled by the kinetic energy initially owned by the particle, and its initial angular speed. Summarizing, the governor can have:

- periodic motions, namely (perpetual) oscillations.

- aperiodic revolutions, where the particle performs infinite rounds on the circle. We will prove the aperiodic revolution is possible in the kinetic case only.

- asymptotic behaviour, where the point unceasingly turns to a position it joins never.

\section{Motion Starting up with Zero Initial Speed}

In this section we are dealing with the Cauchy problem:

$$
\left\{\begin{array}{l}
\ddot{\vartheta}=a \sin \vartheta \cos \vartheta-b \sin \vartheta, \quad a, b>0, \\
\left.\vartheta(0)=\vartheta_{0} \in\right] 0, \pi[ \\
\dot{\vartheta}(0)=0
\end{array}\right.
$$

concerning the motion of a point starting by quiet (akinetic case). Of course the treatment's generality is not reduced if we suppose $\left.\vartheta_{0} \in\right] 0, \pi[$. Performing a first integration we obtain, following the classic Weierstraß approach:

$$
\begin{aligned}
\dot{\vartheta}^{2} & =2 \int_{\vartheta_{0}}^{\vartheta}(a \sin \xi \cos \xi-b \sin \xi) d \xi \\
& =-a\left(\cos \vartheta-\cos \vartheta_{0}\right)\left(\cos \vartheta-\frac{2 b-a \cos \vartheta_{0}}{a}\right) \doteq \Phi(\vartheta) .
\end{aligned}
$$


The problem is defined for all $\vartheta$ values such that:

$$
-a\left(\cos \vartheta-\cos \vartheta_{0}\right)\left(\cos \vartheta-\frac{2 b-a \cos \vartheta_{0}}{a}\right) \geq 0,
$$

whilst the sign for $\dot{\vartheta}$ is given by that of $a \cos \vartheta_{0}-b$, due to the fact that $\sin \vartheta_{0}>0$. The condition (2.3) then induces two different kind of motions, according to either:

or:

$$
\left|\frac{2 b-a \cos \vartheta_{0}}{a}\right| \leq 1
$$

$$
\left|\frac{2 b-a \cos \vartheta_{0}}{a}\right|>1
$$

Let first (2.4) be true. So, the equation $\Phi(\vartheta)=0$ will have four roots in the reference interval $[-\pi, \pi]$, namely two further ones are added to $\vartheta_{0}$ and $-\vartheta_{0}$. Such a condition is clearly equivalent to:

$$
a\left(\cos \vartheta_{0}-1\right) \leq 2 b \leq a\left(\cos \vartheta_{0}+1\right) .
$$

The left hand side inequality in (2.6) is always strictly met, whilst the right hand side one, will be satisfied if:

$$
b \leq \frac{a}{2}\left(\cos \vartheta_{0}+1\right) .
$$

Therefore (2.7) ensures four real roots of $\Phi=0$ in the reference interval. For describing the motion completely, it is necessary to know the couple of further roots induced by the assumption (2.4) with respect to the root $\vartheta_{0}$. Therefore we will analyze three different cases:

$$
\cos \vartheta_{0} \gtreqless \frac{2 b-a \cos \vartheta_{0}}{a} .
$$

The matter is decided by the following oscillation theorem:

Theorem 2.1. If the condition (2.7) holds in the strict sense, i.e.:

$$
b<\frac{a}{2}\left(1+\cos \vartheta_{0}\right)
$$

and, moreover:

$$
a \cos \vartheta_{0}<b,
$$

then a value $\left.\vartheta_{1} \in\right] 0, \vartheta_{0}[$ shall exist such that the motion will be periodic bounded between $\vartheta_{1}$ and $\vartheta_{0}$.

Proof. The (2.7) condition allows to put:

$$
\vartheta_{1}=\arccos \left(\frac{2 b-a \cos \vartheta_{0}}{a}\right),
$$

and, due to (2.8), it there will be: $0<\vartheta_{1}<\vartheta_{0}$. Furthermore $\vartheta_{1}$ is a simple root of $\Phi=0$, and then the thesis follows because the allowed angles shall comply with (2.3). 
In the special cases with the physical parameters $a$ and $b$ such that the $=\operatorname{sign}$ in $(2.7)$ holds, an asymptotic motion will take place.

Theorem 2.2. Under the Theorem 2.1 hypotheses, if:

$$
b=\frac{a}{2}\left(1+\cos \vartheta_{0}\right)
$$

then the particle will point asymptotically towards the south pole of the loop, i.e. $\vartheta_{1}=0$.

Proof. It is easy to see that (2.10) implies $\frac{2 b-a \cos \vartheta_{0}}{a}=1$ and then $\Phi=0$ has a double root in $\vartheta=0$ : the trajectory will reach $\vartheta=0$ in an infinite time.

The angle $\vartheta$ decrease from $\vartheta_{0}$ to $\vartheta_{1}$, ruling to take the minus sign before the root in (1.3), is confirmed by the negative sign of:

$$
\frac{\partial \Phi}{\partial \vartheta}\left(\vartheta_{0}\right)=\sin \vartheta_{0}\left(a \cos \vartheta_{0}-b\right)<0
$$

We can analogously mark the symmetric situation when the inequality in (2.8) is reversed.

Theorem 2.3. If (2.7) and:

$$
a \cos \vartheta_{0}>b .
$$

are both satisfied, then a value $\left.\vartheta_{1} \in\right] \vartheta_{0}, \pi[$ will exist such that the particle moves periodically between $\vartheta_{0}$ and $\vartheta_{1}$.

Proof. The angle $\vartheta_{1}$ is defined as in the previous proof, but now, due to (2.11) we have: $\vartheta_{1}>\vartheta_{0}$. The conclusion follows by the condition (2.3).

We are faced with an asymmetric situation: the retrograde motion will allow asymptotic behaviour towards the south pole of the governor, while an asymptotic motion to the north pole is not possible. Of course it depends on the fact that the initial angular speed is zero. We will prove the existence of both asymptotic motions in the kinetic case.

In order to analyze the degeneration $a \cos \vartheta_{0}=b$, let us note that (2.3) becomes:

$$
-a\left(\cos \vartheta-\cos \vartheta_{0}\right)^{2} \geq 0
$$

and therefore $\vartheta_{0}$ is a stationary solution. This is also confirmed by (2.1) which will give:

$$
\ddot{\vartheta}=a \sin \vartheta\left(\cos \vartheta-\cos \vartheta_{0}\right)
$$

The shown results are founded upon both the assumption (2.7) and the motion reality condition (2.3). If, on the contrary, one assumes the inequality (2.5), then two separate events can occur:

$$
\text { either } \quad \frac{2 b-a \cos \vartheta_{0}}{a}>1, \quad \text { or } \quad \frac{2 b-a \cos \vartheta_{0}}{a}<-1 \text {. }
$$


In the first case we have:

$$
2 b>a\left(\cos \vartheta_{0}+1\right),
$$

then the existence condition (2.3) becomes:

$$
\cos \vartheta \geq \cos \vartheta_{0}
$$

So, we proved the:

Theorem 2.4. If (2.12) is assumed, then the movement, ruled by (2.1) is bounded between the initial equilibrium position $\vartheta_{0}$ and its symmetrical $-\vartheta_{0}$.

If the second occurrence holds in (2.5), namely:

$$
\frac{2 b-a \cos \vartheta_{0}}{a}<-1
$$

we find the motion problem looses its physical meaning because (2.13) is equivalent to $2 b<a\left(\cos \vartheta_{0}-1\right)$, whose validity would allow $b<0$ and, as a consequence, a negative (upwards) gravity acceleration.

The analysis, up to this point, is rather long but crucial for the originality of this paper consisting of the closed form integrations of (2.1). How we will see, if the initial angular speed is zero, the motion will be periodic, except when:

$$
\cos \vartheta_{1}=\frac{2 b-a \cos \vartheta_{0}}{a}=1
$$

as explained by Theorem 2.2 .

\section{The Akinetic Case: Closed form Integration}

We will start under the same assumptions of whichever Theorems 2.1, 2.2 and 2.3. Afterwards the motion equation will be integrated under the Theorem 2.4 assumptions. Following the Theorems 2.1 and 2.3, we will find periodic motion bounded between the anomalies $\vartheta_{0}$ and $\vartheta_{1}$, because the equation $\Phi=0$ has four roots, $\pm \vartheta_{0}, \pm \vartheta_{1}$ in the reference range $[-\pi, \pi]$, whilst for $(2.9) \vartheta_{1}$ is:

$$
\cos \vartheta_{1}=\frac{2 b-a \cos \vartheta_{0}}{a} .
$$

Of course the inequality (2.7) ensures that (3.1) is well defined. The differential equation (2.2) can then be written as:

$$
\dot{\vartheta}=-\sqrt{-a\left(\cos \vartheta-\cos \vartheta_{0}\right)\left(\cos \vartheta-\cos \vartheta_{1}\right)}
$$

and following the Theorem 2.1, we have $\vartheta_{1} \leq \vartheta \leq \vartheta_{0}$, whilst due to the Theorem 2.3, it will be $\vartheta_{0} \leq \vartheta \leq \vartheta_{1}$. 
Theorem 3.1. If the assumptions of Theorem 2.1 are met, so that an oscillation ${ }^{(1)}$ takes place between $\vartheta_{0}$ and $\vartheta_{1}$, with $\vartheta_{0}>\vartheta_{1}>0$, putting:

$$
k^{2}=\frac{2\left(\cos \vartheta_{1}-\cos \vartheta_{0}\right)}{\left(1-\cos \vartheta_{0}\right)\left(1+\cos \vartheta_{1}\right)}, \quad \mu=\frac{2}{\sqrt{\left(1-\cos \vartheta_{0}\right)\left(1+\cos \vartheta_{1}\right)}},
$$

then the motion is found to be:

$$
\vartheta(t)=\arccos \left(\frac{\operatorname{sn}^{2}\left(\frac{\sqrt{a}}{\mu} t, k\right)\left(\cos \vartheta_{0}-\cos \vartheta_{1}\right)-\cos \vartheta_{0}\left(1+\cos \vartheta_{1}\right)}{\operatorname{sn}^{2}\left(\frac{\sqrt{a}}{\mu} t, k\right)\left(\cos \vartheta_{1}-\cos \vartheta_{0}\right)-\left(1+\cos \vartheta_{1}\right)}\right),
$$

$\operatorname{sn}(\bullet, k)$ being the Jacobian elliptic function sine amplitude of the argument $(\bullet)$ and $0<$ $k<1$ its module. The motion period is given by:

$$
T=\frac{4}{\sqrt{a\left(1-\cos \vartheta_{0}\right)\left(1+\cos \vartheta_{1}\right)}} \mathbf{K}(k),
$$

$\mathbf{K}(k)$ being the first kind complete elliptic integral.

Proof. Separating the variables, (3.2) becomes:

$$
t=-\int_{\vartheta_{0}}^{\vartheta} \frac{d \xi}{\sqrt{-a\left(\cos \xi-\cos \vartheta_{0}\right)\left(\cos \xi-\cos \vartheta_{1}\right)}}
$$

Or, putting $\xi=\arccos z$ :

$$
t=\frac{1}{\sqrt{a}} \int_{\cos \vartheta_{0}}^{\cos \vartheta} \frac{d z}{\sqrt{(z-1)(z+1)\left(z-\cos \vartheta_{0}\right)\left(z-\cos \vartheta_{1}\right)}}
$$

We will reduce the elliptic integral (3.6) to the Legendre standard form, founding on the identity proved in [9] chapter 13, vol. 2, pages 307-310:

$$
\int \frac{d z}{\sqrt{\left(z-\alpha_{1}\right)\left(z-\alpha_{3}\right)\left(z-\alpha_{3}\right)\left(z-\alpha_{4}\right)}}=\mu \int \frac{d \varphi}{\sqrt{1-k^{2} \sin ^{2} \varphi}}
$$

where:

$$
\mu=\frac{2}{\sqrt{\left(\alpha_{1}-\alpha_{3}\right)\left(\alpha_{2}-\alpha_{4}\right)}}, \quad k^{2}=\frac{\alpha_{1}-\alpha_{4}}{\alpha_{1}-\alpha_{3}} \cdot \frac{\alpha_{2}-\alpha_{3}}{\alpha_{2}-\alpha_{4}}
$$

$k$ being the module of the first kind elliptic integral, whilst the new variable $\varphi$ (normalizing angle) is defined by:

$$
\sin ^{2} \varphi=\frac{\alpha_{2}-\alpha_{4}}{\alpha_{2}-\alpha_{3}} \cdot \frac{z-\alpha_{3}}{z-\alpha_{4}}
$$

${ }^{(1)}$ When an oscillation is not symmetric, some author like [3] prefers to use the word libration, but we, like [6], will not follow it. 
We shall afterwards remember that [9] $\alpha_{1}>\alpha_{2}>\alpha_{3}>\alpha_{4}$, so we have:

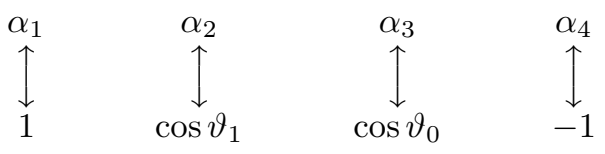

and the integral (3.6) becomes:

$$
t=\frac{1}{\sqrt{a}} \mu \int_{0}^{\arcsin x(\vartheta)} \frac{d \varphi}{\sqrt{1-k^{2} \sin ^{2} \varphi}}
$$

with:

$$
\begin{aligned}
\mu & =\frac{2}{\sqrt{\left(1-\cos \vartheta_{0}\right)\left(1+\cos \vartheta_{1}\right)}}, \\
k^{2} & =\frac{2\left(\cos \vartheta_{1}-\cos \vartheta_{0}\right)}{\left(1-\cos \vartheta_{0}\right)\left(1+\cos \vartheta_{1}\right)}, \\
x(\vartheta) & =\sqrt{\frac{1+\cos \vartheta_{1}}{\cos \vartheta_{1}-\cos \vartheta_{0}} \cdot \frac{\cos \vartheta-\cos \vartheta_{0}}{\cos \vartheta+1}},
\end{aligned}
$$

So we can write (3.8) through the first kind elliptic integral:

$$
t=\frac{\mu}{\sqrt{a}} F(\arcsin x(\vartheta), k)
$$

But:

$$
\mathrm{sn}\left(\frac{\sqrt{a}}{\mu} t, k\right)=\sin (\arcsin x(\vartheta))=x(\vartheta),
$$

then, solving for $\vartheta,(3.3)$ follows.

Let us go to the period, which will be given by the general formula:

$$
T=-2 \int_{\vartheta_{0}}^{\vartheta_{1}} \frac{d \xi}{\sqrt{-a\left(\cos \xi-\cos \vartheta_{0}\right)\left(\cos \xi-\cos \vartheta_{1}\right)}} .
$$

Being now $x\left(\vartheta_{1}\right)=1$, the oscillation period will be given by:

$$
T=2 \frac{\mu}{\sqrt{a}} F\left(\frac{\pi}{2}, k\right)=2 \frac{\mu}{\sqrt{a}} \mathbf{K}(k) .
$$

This leads to (3.4) and the proof is over.

The integration is slightly different for the asymptotic motion due to the Theorem 2.2 assumptions. We enunciate the:

Theorem 3.2. Let the hypotheses of Theorem 2.2 be satisfied, and particularly formula (2.10). Then (2.1) has the solution:

$$
\vartheta(t)=\arccos \left(1-\frac{4\left(1-\cos \vartheta_{0}\right)}{3-\cos \vartheta_{0}+\left(1+\cos \vartheta_{0}\right) \cosh \left(t \sqrt{2 a\left(1-\cos \vartheta_{0}\right)}\right)}\right)
$$


Proof. (2.10) shows that, in force of (2.9), we have $\cos \vartheta_{1}=1$, and then the time equation becomes:

$$
t=-\int_{\vartheta_{0}}^{\vartheta} \frac{d \xi}{\sqrt{-a\left(\cos \xi-\cos \vartheta_{0}\right)(\cos \xi-1)}} .
$$

Now, setting again $\xi=\arccos z$, remembering that $z \geq \cos \vartheta_{0},(3.10)$ gives:

$$
t=\frac{1}{\sqrt{a}} \int_{\cos \vartheta_{0}}^{\cos \vartheta} \frac{d z}{(1-z) \sqrt{(1+z)\left(z-\cos \vartheta_{0}\right)}},
$$

so, if $y=1-z,(3.11)$ is written as:

$$
t=-\frac{1}{\sqrt{a}} \int_{1-\cos \vartheta_{0}}^{1-\cos \vartheta} \frac{d y}{y \sqrt{y^{2}+\left(\cos \vartheta_{0}-3\right) y+2\left(1-\cos \vartheta_{0}\right)}} .
$$

Integral (3.12) is solved, for example in [10]: assuming $c>0$, we have:

$$
\int \frac{d y}{y \sqrt{a y^{2}+b y+c}}=-\frac{1}{\sqrt{c}} \ln \left(\frac{2 \sqrt{c} \sqrt{a y^{2}+b y+c}+b y+c}{y}\right) .
$$

So, after the integration, going back from $y$ to $\vartheta$ and inverting, one will get $\vartheta$ as given by $(3.9)$

The assumptions for the second oscillating case are the same of Theorem 2.3: but the integration will be different, due to the new assumptions' impact on the condition (3.7), which is critical in starting up the analytic machinery exposed in [9].

Theorem 3.3. Let us match the Theorem 2.3 assumptions are true, and specially $a \cos \vartheta_{0}>b$, so that the particle oscillates between $\vartheta_{0}$ and $\vartheta_{1}>\vartheta_{0}$. Putting:

$$
k^{2}=\frac{2\left(\cos \vartheta_{0}-\cos \vartheta_{1}\right)}{\left(1-\cos \vartheta_{1}\right)\left(1+\cos \vartheta_{0}\right)}, \quad \mu=\frac{2}{\sqrt{\left(1-\cos \vartheta_{1}\right)\left(1+\cos \vartheta_{0}\right)}},
$$

the equation (2.1) will have the solution:

$$
\vartheta(t)=\arccos \left(\frac{\left(\cos \vartheta_{0}-\cos \vartheta_{1}\right) \operatorname{sn}^{2}\left(\mathbf{K}(k)-\frac{t}{\mu} \sqrt{a}, k\right)+\cos \vartheta_{1}\left(1+\cos \vartheta_{0}\right)}{\left(1+\cos \vartheta_{0}\right)-\left(\cos \vartheta_{0}-\cos \vartheta_{1}\right) \operatorname{sn}^{2}\left(\mathbf{K}(k)-\frac{t}{\mu} \sqrt{a}, k\right)}\right),
$$

with the period:

$$
T=\frac{4}{\sqrt{a\left(1-\cos \vartheta_{1}\right)\left(1+\cos \vartheta_{0}\right)}} \mathbf{K}(k) .
$$

Proof. The proof is similar to that of the Theorem 3.1, but the identification among the roots following up the [9] procedure is:

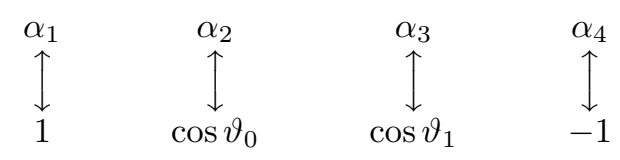


and, as a consequence, the $\mu$ and $k$ expressions have to be accordingly changed.

We now have the residual task of integrating the motion differential equation (2.1) when the governor physical parameters allow only two symmetrical roots of $\Phi=0$; then we assume to be compliant with Theorem 2.4 , namely $2 b>a\left(\cos \vartheta_{0}+1\right)$ having always $\cos \vartheta \geq \cos \vartheta_{0}$ for the movement reality. This will be matter of the:

Theorem 3.4. Under the Theorem 2.4 assumptions, and putting:

$$
p=p\left(a, b, \vartheta_{0}\right)=\frac{2 b-a \cos \vartheta_{0}}{a}>1,
$$

with:

$$
\mu=\sqrt{\frac{2}{p-\cos \vartheta_{0}}}, \quad k^{2}=\frac{(p+1)\left(1-\cos \vartheta_{0}\right)}{2\left(p-\cos \vartheta_{0}\right)},
$$

then the (2.1) differential equation will have a $T$ periodic solution. For $0 \leq t \leq \frac{T}{4}$ and $\frac{3}{4} T \leq t \leq T$, the solution can be written as:

$$
\vartheta(t)=\arccos \left(\frac{\left(1-\cos \vartheta_{0}\right) \operatorname{sn}^{2}\left(\frac{\sqrt{a}}{\mu} t, k\right)+2 \cos \vartheta_{0}}{2-\left(1-\cos \vartheta_{0}\right) \operatorname{sn}^{2}\left(\frac{\sqrt{a}}{\mu} t, k\right)}\right),
$$

while, for $\frac{T}{4} \leq t \leq \frac{3}{4} T$ :

$$
\vartheta(t)=-\arccos \left(\frac{\left(1-\cos \vartheta_{0}\right) \operatorname{sn}^{2}\left(\frac{\sqrt{a}}{\mu} t, k\right)+2 \cos \vartheta_{0}}{2-\left(1-\cos \vartheta_{0}\right) \operatorname{sn}^{2}\left(\frac{\sqrt{a}}{\mu} t, k\right)}\right),
$$

and, in any case, the period is given by:

$$
T=4 \frac{\mu}{\sqrt{a}} \mathbf{K}(k)
$$

Proof. The same integration technique is used as for Theorems 3.1, 3.3, starting from:

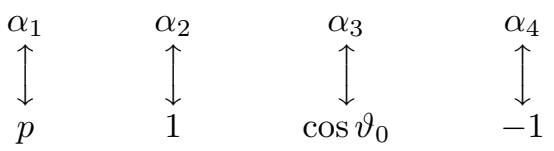

with $\mu$ and $k$ suitable expressions. In fact, the time integral for $\vartheta$ anomalies moving in reverse sense, namely from $\vartheta_{0}$ to $-\vartheta_{0}$, is elliptic:

$$
t=-\frac{1}{\sqrt{a}} \int_{\vartheta_{0}}^{\vartheta} \frac{d \xi}{\sqrt{\left(\cos \xi-\cos \vartheta_{0}\right)(p-\cos \xi)}} .
$$

Following the usual transformation [9], after a little algebra, one gets the period as given by (3.17) and the motion by:

$$
\cos \vartheta(t)=\frac{\left(1-\cos \vartheta_{0}\right) \operatorname{sn}^{2}\left(\frac{\sqrt{a}}{\mu} t, k\right)+2 \cos \vartheta_{0}}{2-\left(1-\cos \vartheta_{0}\right) \operatorname{sn}^{2}\left(\frac{\sqrt{a}}{\mu} t, k\right)} .
$$


So, unlike Theorems 2.1 and 2.3, where the motion takes place between a couple of anomalies within the first quadrant, the inversion of (3.18) requires some caution. By inspection of the right hand side of (3.18), minding the sinus amplitude periodicity, see [2] chapter 6 , section 9 pages $146-147$, we see its period is:

$$
\frac{T}{2}=2 \frac{\mu}{\sqrt{a}} \mathbf{K}(k)
$$

Afterwards, founding upon the elliptic functions' derivatives formulas, see again [2] chapter 6 , section 9 pages $147-148$, this function is found in the range $\left[0, \frac{T}{2}\right]$ to attain its maximum at $t=\frac{T}{4}$, as one can see by (3.18) deriving with respect to $t$ :

$$
\frac{4 \sqrt{a} \sin ^{2} \vartheta_{0} \operatorname{sn}\left(\frac{\sqrt{a}}{\mu} t, k\right) \operatorname{cn}\left(\frac{\sqrt{a}}{\mu} t, k\right) \operatorname{dn}\left(\frac{\sqrt{a}}{\mu} t, k\right)}{\left[\mu\left(2-\left(1-\cos \vartheta_{0}\right) \operatorname{sn}^{2}\left(\frac{\sqrt{a}}{\mu} t, k\right)\right)\right]^{2}},
$$

where cn and dn are the Jacobian elliptic functions cosinus amplitude and delta amplitude respectively. Herefrom we infer the (3.18) monotonicity, built upon both sign and periodicity of the elliptic functions. Its extremal value is 1 , whilst the minimum value - arising for $t=0$ and for $t=\frac{T}{2}$ - is $\cos \vartheta_{0}$ and the (3.18) right hand side qualitative behaviour in the range $\left[0, \frac{T}{2}\right]$ is shown below.

If $0<t<\frac{T}{4}$ the solution of (3.18) will be expressed through the arccos function, whilst for $\frac{T}{4}<t<\frac{T}{2}$ the (3.18) solution will require - arccos. Similarly, on the second halfperiod, where the time equation is:

$$
t-\frac{T}{2}=\frac{1}{\sqrt{a}} \int_{-\vartheta_{0}}^{\vartheta} \frac{d \xi}{\sqrt{\left(\cos \xi-\cos \vartheta_{0}\right)(p-\cos \xi)}}
$$

one can get that the particle will transit through the south pole for $t=\frac{3}{4} T$. Then, if $\frac{T}{2}<t<\frac{3}{4} T$ our inverse function will be $-\arccos$, whilst, in the period last quarter, the inverse will be arccos. This completes the proof.

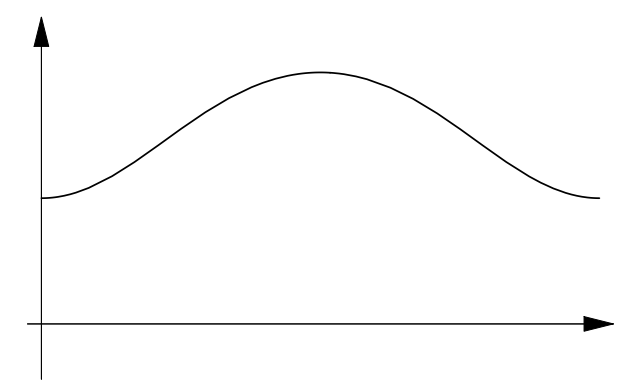

The governor's differential equation generalizes the simple pendulum swinging in a fixed plane, which it is tending to, whenever the plane angular speed is going to zero: $a \rightarrow 0^{+}$. 
The other oscillatory cases treated till now cannot be - according to Theorems 2.1 and 2.3 and to the relevant integrations - a generalization of the fixed plane (and then simple) pendulum: as a matter of fact in those theorems the inequality (2.7) is assumed true, whilst, for $a$ going to zero, one would find $b \leq 0$, namely an unrealistic negative (reverse) gravity, which is against the initial assumptions.

On the contrary, following the assumptions of Theorems 2.4 and 3.4, we see that, for $a \rightarrow 0^{+}$the governor period (3.17), exactly gives back the simple pendulum one:

Corollary 3.1. Under the same assumptions and symbols of Theorems 2.4 and 3.4, then:

$$
\lim _{a \rightarrow 0^{+}} T=\lim _{a \rightarrow 0^{+}} 4 \frac{\mu}{\sqrt{a}} \mathbf{K}(k)=\frac{4}{\sqrt{b}} \mathbf{K}\left(\sin \frac{\vartheta_{0}}{2}\right) .
$$

Proof. First of all let us observe that both $\mu$ and $k$ are continuous functions of $a>0$. From (3.17), (3.13) and (3.14), one easy obtains:

$$
\lim _{a \rightarrow 0^{+}} 4 \frac{\mu}{\sqrt{a}}=\lim _{a \rightarrow 0^{+}} \frac{4}{\sqrt{b-a \cos \vartheta_{0}}}=\frac{4}{\sqrt{b}} .
$$

But the behaviour of $k$ is such that

$$
\lim _{a \rightarrow 0^{+}} k=\lim _{a \rightarrow 0^{+}} \frac{\left(\cos \vartheta_{0}-1\right)\left[a\left(\cos \vartheta_{0}-1\right)-2 b\right]}{4\left(b-a \cos \vartheta_{0}\right)}=\frac{1-\cos \vartheta_{0}}{2} .
$$

The thesis will follow making the limit passage under the integral and remembering a goniometric identity.

The limit we found is in perfect agreement with the simple pendulum period:

$$
T=4 \sqrt{\frac{R}{g}} \mathbf{K}(k),
$$

if one minds that is $b=\frac{g}{R}$ with $g$ gravity acceleration and $R$ circle radius, or pendulum length. Up to this point we highlight that the relevant $\vartheta(t)$ solution versus time (3.15) and (3.16), if $a \rightarrow 0^{+}$will result in the well known formulas for the simple pendulum anomaly as elliptic function of the time.

\section{Non-Zero Initial Speed Motion}

The system dynamics, if the particle angular speed has a non-zero value at $t=0$ (kinetic case), is ruled by the Cauchy problem:

$$
\left\{\begin{array}{l}
\ddot{\vartheta}=a \sin \vartheta \cos \vartheta-b \sin \vartheta, \quad a, b>0, \\
\left.\vartheta(0)=\vartheta_{0} \in\right] 0, \pi[ \\
\dot{\vartheta}(0)=\dot{\vartheta}_{0} \neq 0 .
\end{array}\right.
$$


Following the Weierstraß method, the function whose roots have to be studied becomes:

$$
\dot{\vartheta}^{2}=-a\left(\cos \vartheta-\cos \vartheta_{0}\right)\left(\cos \vartheta-\frac{2 b-a \cos \vartheta_{0}}{a}\right)+\dot{\vartheta}_{0}^{2} \doteq \Phi_{0}(\vartheta),
$$

differing from (2.2) due to the kinetic term ${\dot{\vartheta_{0}}}^{2}$ only, or, going back to the symbols of section $2, \Phi_{0}=\Phi+\dot{\vartheta}_{0}^{2}$.

Because $\Phi_{0}\left(\vartheta_{0}\right)=\dot{\vartheta}_{0}{ }^{2}>0$, the motion reality is now ensured in the neighborhood of $\vartheta_{0}$, which is not the extremal anomaly any more: the existence (if any) of roots of $\Phi_{0}(\vartheta)=0$ will induce: either the periodic motion or complete (and perpetual) aperiodic revolutions; or the asymptotic motion towards one of two poles.

Clearly, recalling that $\Phi(\vartheta)$ is limited, the perpetual aperiodic revolutions are started up by the initial speed values implying $\Phi_{0}(\vartheta)>0$ for each $\vartheta \in[-\pi, \pi]$. Such values, marking the system, will be completely described by the next discussion, built upon the search of an absolute minimum of $\Phi_{0}(\vartheta)$. We will have aperiodic revolutions if and only if the absolute minimum of $\Phi_{0}(\vartheta)$ is strictly greater than zero. First we shall take into account that $\Phi_{0}(\vartheta)$ is an even function of period $2 \pi$ : the minimum will be reached in the interval $[0, \pi]$. Being:

$$
\Phi_{0}^{\prime}(\vartheta)=2(a \cos \vartheta-b) \sin \vartheta
$$

two cases shall be seen:

$$
0<\frac{b}{a} \leq 1 \quad \text { or } \quad \frac{b}{a}>1
$$

\subsection{Case $\frac{b}{a} \leq 1$}

If $b / a \leq 1$, with the nonnegative factor $\sin \vartheta$ for $\vartheta \in[0, \pi]$, the $\Phi_{0}^{\prime}(\vartheta)$ sign, minding (4.3), is ruled by the sign of the quantity $a \cos \vartheta-b$. Then in the interval $[0, \pi]$ we will have that:

and then:

$$
\Phi_{0}^{\prime}(\vartheta) \geq 0 \Longleftrightarrow 0 \leq \vartheta \leq \arccos \left(\frac{b}{a}\right)
$$

$$
\nu \doteq \min _{\vartheta \in[0, \pi]} \Phi_{0}(\vartheta)=\min \left\{\Phi_{0}(0), \Phi_{0}(\pi)\right\}
$$

Furthermore, being:

$$
\begin{aligned}
& \Phi_{0}(0)=\dot{\vartheta}_{0}^{2}+\left(\cos \vartheta_{0}-1\right)\left(a+a \cos \vartheta_{0}-2 b\right), \\
& \Phi_{0}(\pi)={\dot{\vartheta_{0}}}^{2}-\left(\cos \vartheta_{0}+1\right)\left(a-a \cos \vartheta_{0}+2 b\right),
\end{aligned}
$$

it will be easy found that:

$$
\Phi_{0}(\pi)=\Phi_{0}(0)-4 b \Rightarrow \Phi_{0}(\pi)<\Phi_{0}(0) .
$$

So we proved that:

$$
\nu=\Phi_{0}(\pi)={\dot{\vartheta_{0}}}^{2}-\left(\cos \vartheta_{0}+1\right)\left(a-a \cos \vartheta_{0}+2 b\right) .
$$


The first of the motion's characterization in the kinetic case is established by the:

Theorem 4.1. If $b / a \leq 1$, then the motion ruled by (4.2) will be revolutive if and only if the initial speed $\dot{\vartheta}_{0}$ satisfies the inequality:

$$
\dot{\vartheta}_{0}^{2}>\left(\cos \vartheta_{0}+1\right)\left(a-a \cos \vartheta_{0}+2 b\right) .
$$

Proof. The (4.5) inequality ensures that the minimum of $\Phi_{0}(\vartheta)$, evaluated in (4.4), is strictly greater than zero. This will prevent from $\Phi_{0}(\vartheta)$ going to zero, excluding both revolutive and asymptotic motions: as a consequence, the point will carry revolutions on the rotating plane.

Let us show a plot of $\Phi_{0}(\vartheta)$ for: $a=2, b=1, \vartheta_{0}=\pi / 4, \dot{\vartheta}_{0}=2.4$.

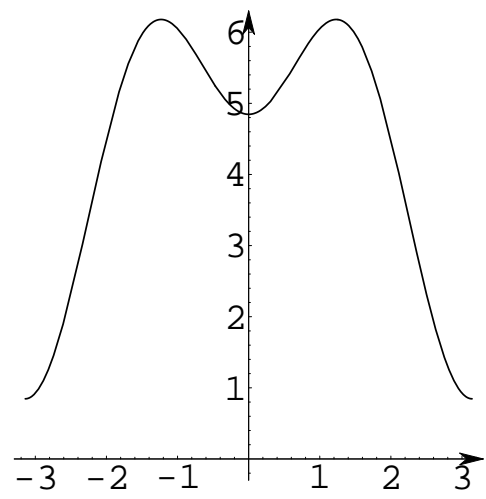

It is possible to highlight specifically the $\Phi_{0}$ behaviour according to the Theorem 4.1, and this will be carried out in the next corollary, in order to integrate the motion equation explicitly, what will be done at the next section under the Theorem 4.1 assumptions.

Corollary 4.1. Under the assumptions of the Theorem 4.1 , two numbers $p_{+}, p_{-}>1$ exist such that:

$$
\Phi_{0}(\vartheta)=-a\left(\cos \vartheta-p_{+}\right)\left(\cos \vartheta+p_{-}\right)
$$

Proof. The $\Phi_{0}(z)$ discriminant, thinking $\Phi_{0}(z)$ as a second degree polynomial in $z=\cos \vartheta$ is:

$$
\frac{\Delta}{4}=b^{2}-2 a b \cos \vartheta_{0}+a^{2} \cos ^{2} \vartheta_{0}+a \dot{\vartheta}_{0}^{2} .
$$

But, in force of (4.5), by (4.6), one gets:

$$
\begin{aligned}
\frac{\Delta}{4} & >b^{2}-2 a b \cos \vartheta_{0}+a^{2} \cos ^{2} \vartheta_{0}+a\left(\cos \vartheta_{0}+1\right)\left(a-a \cos \vartheta_{0}+2 b\right) \\
& =(a+b)^{2}>0
\end{aligned}
$$


and then, for the major root $p_{+}$:

$$
\begin{aligned}
p_{+} & =\frac{b+\sqrt{b^{2}-2 a b \cos \vartheta_{0}+a^{2} \cos ^{2} \vartheta_{0}+a{\dot{\vartheta_{0}}}^{2}}}{a} \\
& >\frac{b+a+b}{a}=1+2 \frac{b}{a}>1 .
\end{aligned}
$$

And similarly:

$$
\begin{aligned}
p_{-} & =\frac{b-\sqrt{b^{2}-2 a b \cos \vartheta_{0}+a^{2} \cos ^{2} \vartheta_{0}+a \dot{\vartheta}_{0}^{2}}}{a} \\
& <\frac{b-(a+b)}{a}=-1 .
\end{aligned}
$$

This completes the proof.

The (4.5) condition selects whether asymptotic behaviours are possible or not: if the equation is satisfied:

$$
\dot{\vartheta}_{0}^{2}=\left(\cos \vartheta_{0}+1\right)\left(a-a \cos \vartheta_{0}+2 b\right),
$$

then we find a double root for $\Phi_{0}$ in $\pi$, and this does mean asymptotic motion, see [1]. The point will tend asymptotically to the north pole, i.e. $\vartheta=\pi$, because, by (4.7) we now obtain:

$$
\Phi_{0}(\vartheta)=-a(\cos \vartheta+1)\left(\cos \vartheta-\frac{a+2 b}{a}\right),
$$

remembering that $\frac{a+2 b}{a}>1$.

Finally, we have to analyze the system periodic motions. Let us make the assumption:

$$
\dot{\vartheta}_{0}^{2}<\left(\cos \vartheta_{0}+1\right)\left(a-a \cos \vartheta_{0}+2 b\right) .
$$

Theorem 4.2. If the angular speed $\dot{\vartheta}_{0}$ meets the inequality (4.8), then the motion system will be periodic between the symmetric anomalies $\vartheta_{1}$ and $-\vartheta_{1}$ if the inequality:

$$
\dot{\vartheta}_{0}^{2}>\left(1-\cos \vartheta_{0}\right)\left(a+a \cos \vartheta_{0}-2 b\right) .
$$

holds. If the reverse is true:

$$
\dot{\vartheta}_{0}^{2}<\left(1-\cos \vartheta_{0}\right)\left(a+a \cos \vartheta_{0}-2 b\right),
$$

then the motion will be periodic too, but the oscillations will take place between the asymmetric anomalies $\vartheta_{1}$ e $\vartheta_{2}$.

Proof. If (4.9) holds, then $\Phi=0$ will have two roots only in $[-\pi, \pi]$ and they will be necessarily opposite. Then the motion will take place between them, passing through the 
south pole. If, on the contrary, (4.10) holds, then $\Phi_{0}$ will have its four zeros in $[-\pi, \pi]$, and the motion will be between those zeros belonging to $[0, \pi]$, with $\vartheta_{0}>0$.

The $\Phi_{0}$ behaviour versus $\vartheta$ is shown by the following plots: whose the first has $a=3$, $b=1, \vartheta_{0}=\pi / 4$ so that (4.9) is satisfied; for the second is $a=4.3, b=1, \vartheta_{0}=\pi / 4$, referring to the situation $(4.10)$.
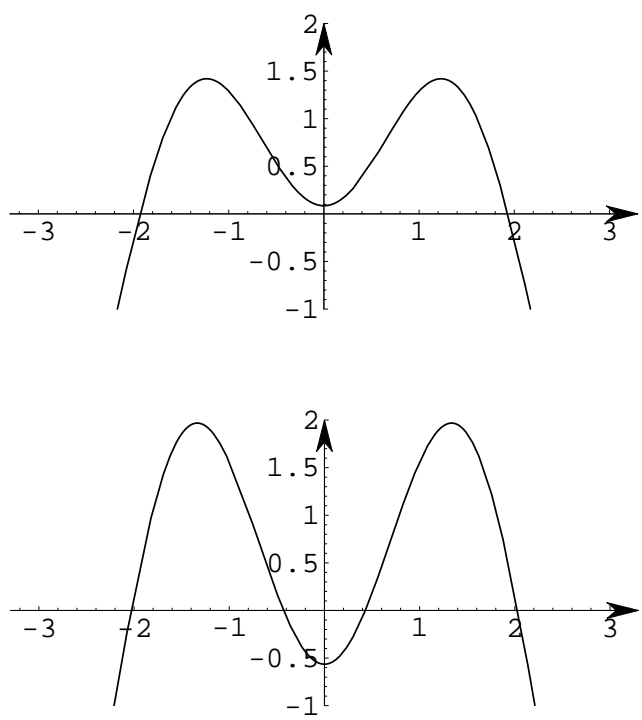

Finally, the degenerate case:

$$
\dot{\vartheta}_{0}^{2}=\left(1-\cos \vartheta_{0}\right)\left(a+a \cos \vartheta_{0}-2 b\right),
$$

has to be considered: here $\Phi_{0}=0$ has a double root at the origin, and then the particle, after having passed one time through the positive root of $\Phi_{0}$, will asymptotically move towards the south pole, i.e. $\vartheta=0$. The last is well founded on the fact that (4.11) allows to write $\Phi_{0}$ as:

$$
\Phi_{0}=a(1-\cos \vartheta)\left(\cos \vartheta-\frac{2 b-a}{a}\right)
$$

where the assumption $\frac{b}{a} \leq 1$ implies $\frac{2 b-a}{a} \leq 1$.

\subsection{Case $\frac{b}{a}>1$}

In this case $\Phi_{0}$ will have mostly two symmetrical roots. By (4.3) we see that $\Phi_{0}$ decreases in $[0, \pi]$ and the minimum is assumed at $\pi$. We have then the:

Theorem 4.3. If $b / a>1$, the motion, described as Cauchy problem (4.2), will be revolutive if and only if the initial speed $\dot{\vartheta}_{0}$ complies with (4.5). If, on the contrary, (4.7) 
is true, the point will tend asymptotically to the north pole; finally in the case (4.8), we will have symmetric oscillations.

The $\Phi_{0}$ plot versus $\vartheta$ for the symmetric oscillating case is given by the figure:

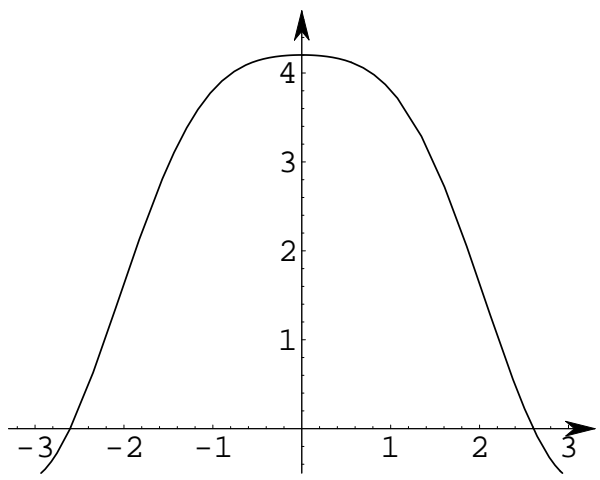

\section{The Kinetic Case: Closed form Integration}

We are now integrating the motion differential equation (4.1) (kinetic case), referring to [9], restricting ourselves to the revolutive case and to the asymptotic occurrences where either (4.7) or (4.11) are met. In fact in the kinetic cases where the behavior is periodic, at the particle culmination, say at time $t_{1}$, the akinetic case (see section 3 ) takes place again.

Let us start, assuming to be in the case, $\frac{b}{a} \leq 1$ (Theorem 4.1 ), chiefly the inequality (4.5) is satisfied. Founding on the corollary 4.1, the correspondence with the [9] notations is:

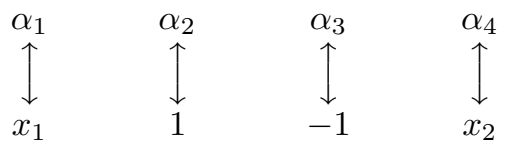

We can so formulate the revolutive explicit integration, but the change $\vartheta=\arccos z$, used for the Theorems 3.1, 3.3 and 3.4, is possible, in order to invert, only if $-\pi<\vartheta<\pi$.

Theorem 5.1. Under the same assumptions of the Theorem 4.1, if we suppose $\dot{\vartheta}_{0}>0$, putting:

$$
\mu=\frac{2}{\sqrt{\left(1+x_{1}\right)\left(1-x_{2}\right)}}, \quad k^{2}=\frac{2\left(x_{1}-x_{2}\right)}{\left(1+x_{1}\right)\left(1-x_{2}\right)},
$$

where $x_{1}$ and $x_{2}$ are the roots of the equation $\Phi_{0}(x)=0$, thought as polynomial of $x=\cos \vartheta$, the solution of the differential equation (4.1), for $\vartheta$ between $\vartheta_{0}$ and $\pi$, as given 
by:

$$
\vartheta(t)=\arccos \left(\frac{x_{2}-1-2 x_{2} \operatorname{sn}^{2}\left(\frac{\sqrt{a}}{\mu} t-F\left(\arcsin x\left(\vartheta_{0}\right), k\right), k\right)}{1-x_{2}-2 \operatorname{sn}^{2}\left(\frac{\sqrt{a}}{\mu} t-F\left(\arcsin x\left(\vartheta_{0}\right), k\right), k\right)}\right),
$$

where:

$$
x(\vartheta)=\sqrt{\frac{x_{2}-1}{2} \frac{1+\cos \vartheta}{x_{2}-\cos \vartheta}} .
$$

Proof. Performing the change of variables $\xi=\arccos \vartheta$, we can write the time equation as:

$$
t=\frac{1}{\sqrt{a}} \int_{\cos \vartheta_{0}}^{\cos \vartheta} \frac{d z}{\sqrt{\left(z-x_{1}\right)(z-1)(z+1)\left(z-x_{2}\right)}}
$$

and ever since we can perform the integration following the [9] approach.

Wishing to integrate the motion equation for $\vartheta>\pi$, one shall correctly invert the cosinus function: e.g. if $\vartheta \in[\pi, 2 \pi]$ the inverse of $z=\cos \vartheta$ is $\vartheta=2 \pi-\arccos z$, and so on.

Under the assumptions of Theorem 4.3, the integration does not differ from that of Theorems 3.1, 3.3, 3.4.

Now let us see two asymptotic motions, starting with that which is pointing towards the north pole.

Theorem 5.2. Let $b \leq a$ and the (4.7) equation. Then, and this is not against the generality, if $\dot{\vartheta}_{0}>0$, the $(4.1)$ has the solution:

$$
\vartheta(t)=\arccos \left(\frac{8(a+b) e^{2 \sqrt{a+b} t}\left(1+\cos \vartheta_{0}\right)}{d(t)}-1\right)
$$

where:

$$
\begin{aligned}
d(t)= & 2 a+3 b+2(2 a+b) e^{2 \sqrt{a+b} t}+(2 a+3 b) e^{4 \sqrt{a+b} t}-(2 a+b)\left(e^{2 \sqrt{a+b} t}-1\right)^{2} \cos \vartheta_{0} \\
& +2 \sqrt{a+b}\left(e^{4 \sqrt{a+b} t}-1\right) \sqrt{\left(\cos \vartheta_{0}-1\right)\left(a \cos \vartheta_{0}-a-2 b\right)} .
\end{aligned}
$$

Proof. In this case the time equation, after some substitutions, like those explained at 3.2 proof, is:

$$
t=-\frac{1}{\sqrt{a}} \int_{1+\cos \vartheta_{0}}^{1+\cos \vartheta} \frac{d x}{x \sqrt{x^{2}-\frac{2(2 a+b)}{2} x+\frac{4(a+b)}{a}}}
$$

and through the indefinite integration used in the proof of Theorem 3.2, $\vartheta$ is successively obtained, giving (5.2).

Finally, let us go to integrate the asymptotic motion towards the south pole: for simplicity we will assume $\dot{\vartheta}_{0}<0$. 
Theorem 5.3. Let it be $b \leq a$ and (4.11) true. Then, assuming - what is not against the generality $-\dot{\vartheta}_{0}<0$, the (4.1) has the solution:

$$
\vartheta(t)=\arccos \left(\frac{n_{1}(t)+n_{2}(t)}{d_{1}(t)+d_{2}(t)}\right),
$$

where:

$$
\begin{aligned}
\left(1-\cos ^{2} \vartheta_{0}\right) n_{1}(t)= & 4 b^{2}\left(\cos \vartheta_{0}-1\right)^{2}+8(2 a-3 b) e^{2 \sqrt{a-b} t}\left(\cos \vartheta_{0}-1\right)\left(2 a-3 b+(2 a-b) \cos \vartheta_{0}\right) \\
+ & 4 e^{4 \sqrt{a-b} t}\left[3 b-2 a+(b-2 a) \cos \vartheta_{0}\right]^{2} \\
+ & 16 e^{2 \sqrt{a-b} t} \cos \frac{\vartheta_{0}}{2} \sqrt{2(a-b)\left(a-2 b+a \cos \vartheta_{0}\right)} \\
\times & {\left[(2 a-3 b)\left(\cos \vartheta_{0}-1\right)+e^{2 \sqrt{a-b} t}\left(2 a-3 b+(2 a-b) \cos \vartheta_{0}\right)\right] } \\
n_{2}(t)= & 8(a-b) e^{4 \sqrt{a-b} t}\left(a-2 b+a \cos \vartheta_{0}\right) \cot ^{2} \frac{\vartheta_{0}}{2} \csc ^{2} \frac{\vartheta_{0}}{2}
\end{aligned}
$$

while:

$$
\begin{aligned}
\left(1-\cos ^{2} \vartheta_{0}\right) d_{1}(t)= & 4 b^{2}\left(\cos \vartheta_{0}-1\right)^{2}-8(2 a-b) e^{2 \sqrt{a-b} t}\left(\cos \vartheta_{0}-1\right)\left(2 a-3 b+(2 a-b) \cos \vartheta_{0}\right) \\
+ & 4 e^{4 \sqrt{a-b} t}\left[3 b-2 a+(b-2 a) \cos \vartheta_{0}\right]^{2} \\
+ & 16 e^{2 \sqrt{a-b} t} \cos \frac{\vartheta_{0}}{2} \sqrt{2(a-b)\left(a-2 b+a \cos \vartheta_{0}\right)} \\
& \times\left[(b-2 a)\left(\cos \vartheta_{0}-1\right)+e^{2 \sqrt{a-b} t}\left(2 a-3 b+(2 a-b) \cos \vartheta_{0}\right)\right]
\end{aligned}
$$

and finally:

$$
d_{2}(t)=8(a-b) e^{4 \sqrt{a-b} t}\left(a-2 b+a \cos \vartheta_{0}\right) \cot ^{2} \frac{\vartheta_{0}}{2} \csc ^{2} \frac{\vartheta_{0}}{2}
$$

\section{Conclusions}

In this final section we show a graphical overview, on the $(\vartheta, t)$ plane, of the testsolutions we obtained applying the final formulas for reasonable values of $a, b, \vartheta_{0}, \dot{\vartheta}_{0}$ compliant with the Theorems 3.1, 3.2, 3.3, 3.4, 5.1, 5.2 and 5.3. We recommend the highest caution using the arccos function specially with revolutive motion or when the particle symmetrically oscillates between the anomalies $\vartheta_{0}$ and $-\vartheta_{0}$ : indeed the found formulas are true for angles enclosed in the reference range $[-\pi, \pi]$. A slight change would be necessary for inverting the cos function for anomalies not included in it.

Further control tests have been accomplished using a numerical high performance method [8]: a complete and minute overlap has been always found. 


\subsection{An example from Theorem 3.1}

Let us consider a test (oscillating, akinetic) case with $a=4, b=1, \vartheta_{0}=\frac{\pi}{2}$, so it will be also $\vartheta_{1}=\frac{\pi}{3}$. All these data are compliant with the Theorems 2.1 and 3.1. The exact solution, special case of (3.3) is:

$$
\vartheta(t)=\arccos \left(\frac{\operatorname{sn}^{2}\left(\sqrt{\frac{3}{2}} t, \frac{2}{3}\right)}{\operatorname{sn}^{2}\left(\sqrt{\frac{3}{2}} t, \frac{2}{3}\right)-3}\right)
$$

which has been plotted below:

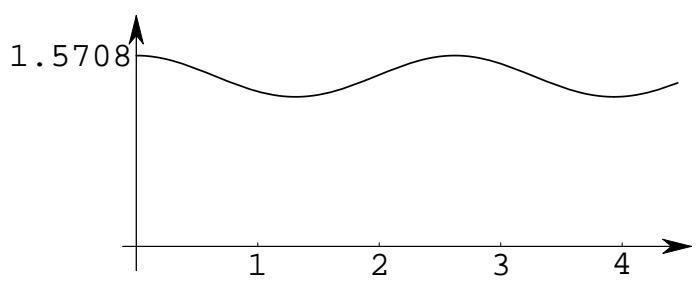

The oscillating behaviour between the limiting angles, corresponding to the (perpetual, for the frictionless assumption) sequel of maxima and minima anomalies, is clearly displayed. The shape of the line apparently resembles a sinusoidal line, but it is of deeply different nature! Finally the oscillatory period $T=2 \sqrt{\frac{2}{3}} \mathbf{K}\left(\frac{2}{3}\right) \approx 3.31328$ is given by equation (3.4).

\subsection{An example from Theorem 3.2}

We tackle an asymptotic motion towards the south pole with $a=1$ and $\vartheta_{0}=\frac{\pi}{3}$, then (2.10) gives $b=\frac{3}{4}$. The (1.1) solution in this special case is:

$$
\vartheta(t)=\arccos \left(\frac{1+3 \cosh t}{5+3 \cosh t}\right),
$$

whose plot, clearly explaining the asymptotic motion character, is shown below:

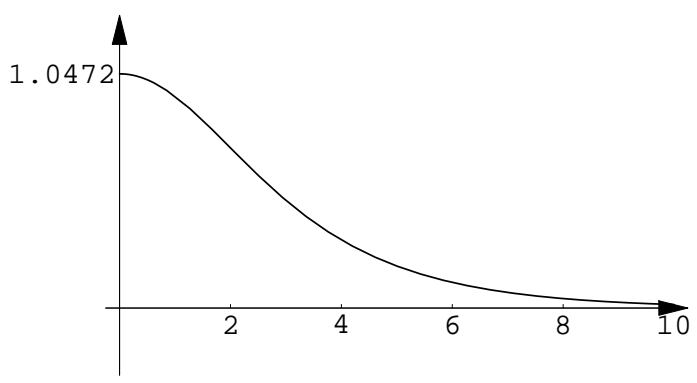




\subsection{An example from Theorem 3.3}

Choosing $a=6, b=2$ and $\vartheta_{0}=\frac{\pi}{3}$, according to the assumptions of Theorems 2.3 and 3.3 with reference to the inequality (2.11), we obtain for (1.1) the solution:

$$
\vartheta(t)=\arccos \left(\frac{3+4 \mathrm{sn}^{2}\left(\mathbf{K}\left(\frac{8}{15}\right)-\frac{1}{2} \sqrt{\frac{15}{2}} t, \frac{8}{15}\right)}{18-4 \operatorname{sn}^{2}\left(\mathbf{K}\left(\frac{8}{15}\right)-\frac{1}{2} \sqrt{\frac{15}{2}} t, \frac{8}{15}\right)}\right) .
$$

whose period is:

$$
T=4 \sqrt{\frac{2}{15}} \mathbf{K}\left(\frac{8}{15}\right) \approx 2.75087 .
$$

The solution has been plotted below, and its oscillating character is clearly displayed. Note that $\vartheta_{1}=\arccos \frac{1}{6} \approx 1.40335$.

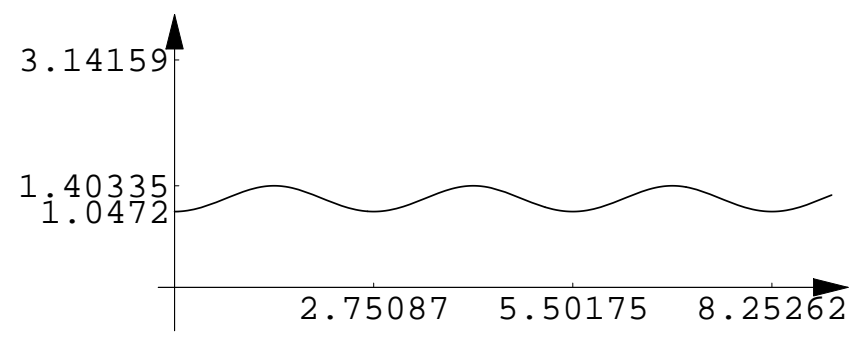

\subsection{An example from Theorem 3.4}

Let us tackle when (Theorems 2.4 and 3.4) the particle moves symmetrically and periodically between opposite anomalies. The test case has been carried out selecting $a=2, b=2$ and $\vartheta_{0}=\frac{\pi}{2}$. The period will be computed as $T=2 \sqrt{2} \mathbf{K}\left(\frac{3}{4}\right) \approx 6.09955$.

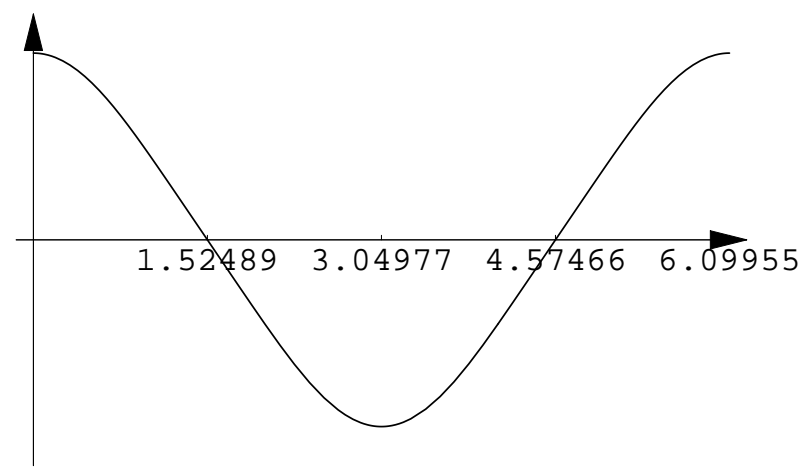




\subsection{An example from Theorem 5.1}

Under the assumptions of Theorems 4.1 and 5.1, infinite revolutions will occur, and the inversion - obtained in (5.1) - is correct in a suitable neighborhood of the starting time. Let us choose $a=2, b=1, \vartheta_{0}=\frac{\pi}{2}$ and $\dot{\vartheta}_{0}=2 \sqrt{3}$ being compliant with the inequality (4.5). The (1.1) solution in this particular case is:

$$
\vartheta(t)=\arccos \left(\frac{4 \operatorname{sn}^{2}\left(\sqrt{6} t-F\left(\frac{\pi}{3}, \frac{5}{6}\right), \frac{5}{6}\right)-3}{3-2 \operatorname{sn}^{2}\left(\sqrt{6} t-F\left(\frac{\pi}{3}, \frac{5}{6}\right), \frac{5}{6}\right)}\right)
$$

whose plot - which apparently resembles a straight line - explains the fact that - in this case of revolvements - the anomaly grows up indefinitely (secular build - up).

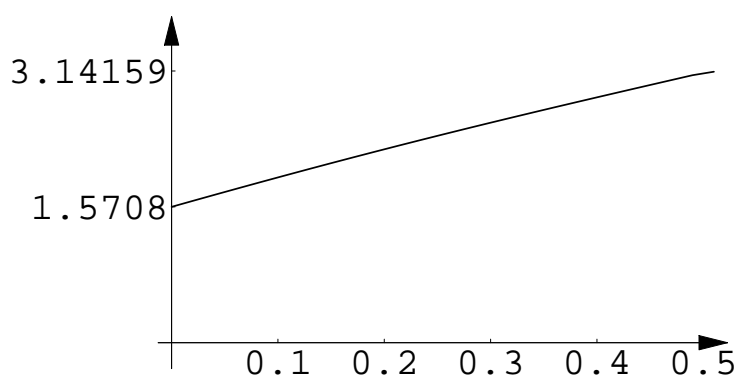

\subsection{An example from Theorem 5.2}

The motion asymptotic nature displayed by the Theorem 5.2 - as it has been shown is induced by the peculiar mixing of the system physical characteristics with the motion initial conditions. Choosing $a=3, b=1$ and $\vartheta_{0}=\frac{\pi}{2}$, then (4.7) will imply $\dot{\vartheta}_{0}$, so that the solution for (1.1) is:

$$
\vartheta(t)=\arccos \left(\frac{32(9+4 \sqrt{5}) e^{4 t}}{1+14(9+4 \sqrt{5}) e^{4 t}+(161+72 \sqrt{5}) e^{8 t}}-1\right)
$$

whose plot, clearly explaining the asymptotic motion to the north pole of the round, is shown below. 


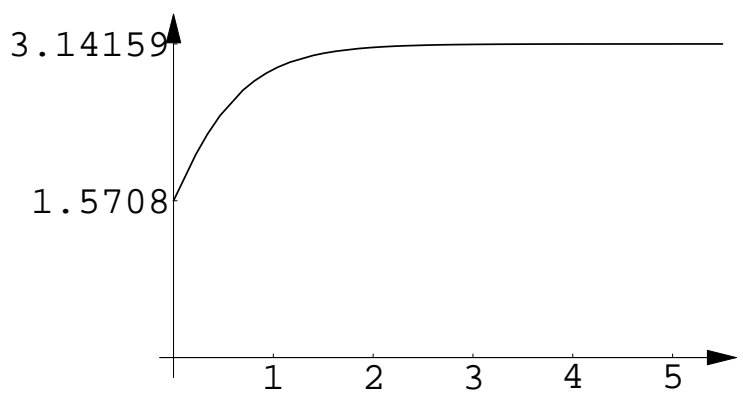

\subsection{An example from Theorem $\mathbf{5 . 3}$}

Let us conclude, showing the case of the Theorem 5.3. Having taken $a=3, b=1$ and $\vartheta_{0}=\frac{\pi}{2},(4.11)$ provides $\dot{\vartheta}_{0}$ and the solution is:

$$
\vartheta(t)=\arccos \left(\frac{\left(e^{2 \sqrt{2} t}-1\right)\left((17+12 \sqrt{2}) e^{2 \sqrt{2} t}-1\right)}{1+10(3+2 \sqrt{2}) e^{2 \sqrt{2} t}+(17+12 \sqrt{2}) e^{4 \sqrt{2} t}}\right),
$$

whose plot shows the motion towards the south pole of the circular trajectory.

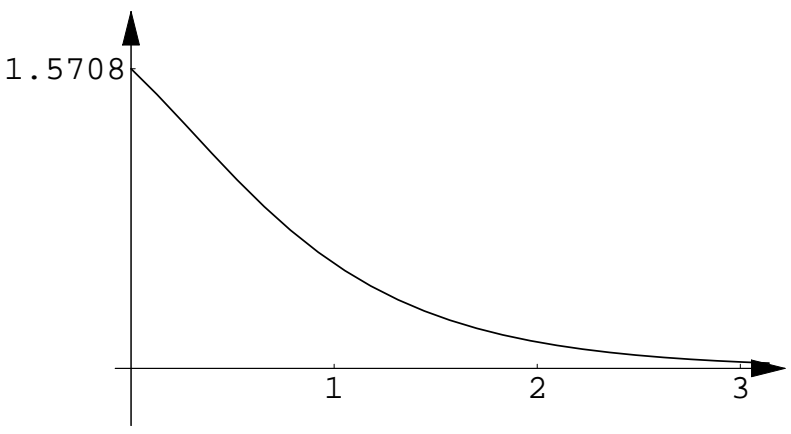

About this solution one can remark that $\dot{\vartheta}_{0}<0$ whilst the plot in 6.2 had horizontal its tangent at the origin for being the particle starting by the rest.

\section{References}

[1] Agostinelli C., Pignedoli A., Meccanica Razionale, 1(1978), Bologna.

[2] Davis H. T., Introduction to Nonlinear Differential and Integral Equations, New York, 1962.

[3] Elipe A., Abad A., Deprit A., A resonance model on a cylinder, Int. J. of Non-Linear Mech., 36(2001), 693-708. 
[4] Jordan D. W., Smith P., Nonlinear Ordinary Differential Equations, Oxford, 1999.

[5] Minorsky N., Introduction to Nonlinear Mechanics, Ann Arbor, 1947.

[6] Pars L. A., A Treatise on Analytical Dynamics, New York, 1965. Reprint Woodbridge, 1979.

[7] Roy L., Cours de Mècanique Rationelle, Tome I, Paris, 1945.

[8] Schwalbe D., Wagon S., VisualDSolve. Visualizing Differential Equations with Mathematica, New York, 1997.

[9] Staff of the Bateman Manuscript Project (Erdélyi A., Magnus W., Oberhettinger F., Tricomi F. G.): Higher Transcendental Functions, New York, 1953.

[10] Zwillinger D., ed., CRC Standard Mathematical Tables, 30 ${ }^{\text {th }}$ edition, CRC Press 1996.

E-mail: giovannimingari@libero.it

Dipartimento di matematica per le scienze economiche e sociali, viale Filopanti, 540127 Bologna Italy.

E-mail: dritelli@economia.unibo.it 